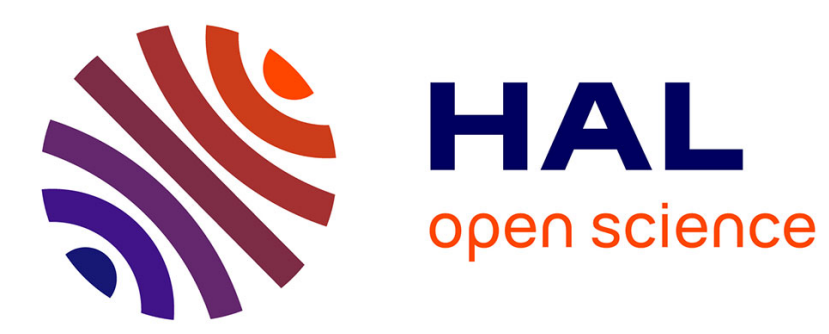

\title{
Twenty Two Parameter Deformations of the Twelfth Peregrine Breather Solutions to the NLS Equation
}

Pierre Gaillard, Mickaël Gastineau

\section{To cite this version:}

Pierre Gaillard, Mickaël Gastineau. Twenty Two Parameter Deformations of the Twelfth Peregrine Breather Solutions to the NLS Equation. Advances in Research, 2016, 7 (2), pp.1-11. 10.9734/AIR/2016/25636 . hal-01299469

\section{HAL Id: hal-01299469 \\ https://hal.science/hal-01299469}

Submitted on 7 Apr 2016

HAL is a multi-disciplinary open access archive for the deposit and dissemination of scientific research documents, whether they are published or not. The documents may come from teaching and research institutions in France or abroad, or from public or private research centers.
L'archive ouverte pluridisciplinaire HAL, est destinée au dépôt et à la diffusion de documents scientifiques de niveau recherche, publiés ou non, émanant des établissements d'enseignement et de recherche français ou étrangers, des laboratoires publics ou privés. 


\title{
Families of deformations of the twelfth Peregrine breather solutions to the NLS equation with twenty two parameters.
}

${ }^{+}$Pierre Gaillard, ${ }^{\times}$Mickaël Gastineau

+ Université de Bourgogne,

9 Av. Alain Savary, Dijon, France : Dijon, France :

e-mail: Pierre.Gaillard@u-bourgogne.fr,

× IMCCE, Observatoire de Paris, PSL Research University, CNRS, Sorbonne Universités, UPMC Univ Paris 06, Univ. Lille,

77 Av. Denfert-Rochereau, 75014 Paris, France :

e-mail: Mickael.Gastineau@obspm.fr

April 2, 2016

\begin{abstract}
The twelfth Peregrine breather $\left(P_{12}\right.$ breather) solution to the focusing one dimensional nonlinear Schrödinger equation (NLS) with its twenty two real parameters deformations solutions to the NLS equation are explicitly constructed here. New families of quasi-rational solutions of the NLS equation in terms of explicit quotients of polynomials of degree 156 in $x$ and $t$ by a product of an exponential depending on $t$ are obtained. The patterns of the modulus of these solutions in the $(x ; t)$ plane, in function of the different parameters are studied in details.
\end{abstract}

PACS : 35Q55, 37K10.

Keywords : NLS equation, wronskians, Peregrine breather, rogue waves. 


\section{Introduction}

Since the first resolution of the one dimensional focusing nonlinear Schrödinger equation (NLS) in 1972 by Zakharov and Shabat using the inverse scattering method [1, 2], a lot of studies have been carried out. The first quasi-rational solution to NLS equation was constructed in 1983 by Peregrine [3]. Akhmediev, Eleonskii and Kulagin obtained the twophase almost periodic solution to the NLS equation and obtained the first higher order analogue of the Peregrine breather $[4,5]$ in 1986 ; other families of higher order 3 and 4 were constructed in a series of articles by Akhmediev et al. [6, 7], using Darboux transformations.

Since the beginning of the years 2010, many works were published using various methods, in particular a formulation by means of wronskians was given in [8]. Recently, in 2013, it was found in [9], solutions expressed in terms of determinants of order $2 N$ depending on $2 N-2$ real parameters. A new representation has been found as a ratio of a determinant of order $N+1$ by another one of order $N$ by Ling and Zhao in [10]. Very recently in 2014, another approach has been given in [11] using a dressing method where the solutions are expressed as the quotient of a determinant of order $N+1$ by another one of order $N$.

In the present work, we construct new solutions to the focusing one dimensional nonlinear Schrödinger equation which appear as deformations of the (analogue) Peregrine breather of order 12 with 22 real parameters. These solutions are completely expressed as a quotient of two polynomials of degree 156 in $x$ and $t$ by an exponential de- pending on $t$. Because of the length of the solutions in terms of polynomials of $x$ and $t$, we cannot give them in this text; only plots in the $(x, t)$ plane of the modulus of the solutions to analyze the evolution of the solutions in function of the different parameters are presented in details here.

\section{Determinant represen- tation of solutions to NLS equation}

In $[16,32]$ the following result have been proved :

Theorem 2.1 The function $v$ defined by

$v(x, t)=\frac{\operatorname{det}\left(\left(n_{j k)_{j, k \in[1,2 N]}}\right)\right.}{\operatorname{det}\left(\left(d_{j k)_{j, k \in[1,2 N]}}\right)\right.} e^{(2 i t-i \varphi)}$

is a quasi-rational solution to the NLS equation

$$
i v_{t}+v_{x x}+2|v|^{2} v=0,
$$

where

$$
\begin{aligned}
& n_{j 1}=f_{j, 1}(x, t, 0), \\
& n_{j k}=\frac{\partial^{2 k-2} f_{j, 1}}{\partial \epsilon^{2 k-2}}(x, t, 0), \\
& n_{j N+1}=f_{j, N+1}(x, t, 0), \\
& n_{j N+k}=\frac{\partial^{2 k-2} f_{j, N+1}}{\partial \epsilon^{2 k-2}}(x, t, 0), \\
& d_{j 1}=g_{j, 1}(x, t, 0), \\
& d_{j k}=\frac{\partial^{2 k-2} g_{j, 1}}{\partial \epsilon^{2 k-2}}(x, t, 0), \\
& d_{j N+1}=g_{j, N+1}(x, t, 0), \\
& d_{j N+k}=\frac{\partial^{2 k-2} g_{j, N+1}}{\partial \epsilon^{22-2}}(x, t, 0), \\
& 2 \leq k \leq N, 1 \leq j \leq 2 N
\end{aligned}
$$


The functions $f$ and $g$ are defined for The parameters $e_{\nu}$ are given by

$1 \leq k \leq N$ by :

$$
\begin{aligned}
& f_{4 j+1, k}=\gamma_{k}^{4 j-1} \sin A_{k}, \\
& f_{4 j+2, k}=\gamma_{k}^{4 j} \cos A_{k}, \\
& f_{4 j+3, k}=-\gamma_{k}^{4 j+1} \sin A_{k}, \\
& f_{4 j+4, k}=-\gamma_{k}^{4 j+2} \cos A_{k}, \\
& f_{4 j+1, N+k}=\gamma_{k}^{2 N-4 j-2} \cos A_{N+k}, \\
& f_{4 j+2, N+k}=-\gamma_{k}^{2 N-4 j-3} \sin A_{N+k}, \\
& f_{4 j+3, N+k}=-\gamma_{k}^{2 N-4 j-4} \cos A_{N+k}, \\
& f_{4 j+4, k}=\gamma_{k}^{2 N-4 j-5} \sin A_{N+k}, \\
& g_{4 j+1, k}=\gamma_{k}^{4 j-1} \sin B_{k}, \\
& g_{4 j+2, k}=\gamma_{k}^{4 j} \cos B_{k}, \\
& g_{4 j+3, k}=-\gamma_{k}^{4 j+1} \sin B_{k}, \\
& g_{4 j+4, k}=-\gamma_{k}^{4 j+2} \cos B_{k}, \\
& g_{4 j+1, N+k}=\gamma_{k}^{2 N-4 j-2} \cos B_{N+k}, \\
& g_{4 j+2, N+k}=-\gamma_{k}^{2 N-4 j-3} \sin B_{N+k}, \\
& g_{4 j+3, N+k}=-\gamma_{k}^{2 N-4 j-4} \cos B_{N+k}, \\
& g_{4 j+4, N+k}=\gamma_{k}^{2 N-4 j-5} \sin B_{N+k},
\end{aligned}
$$

$$
\begin{aligned}
& e_{j}=i \sum_{k=1}^{N-1} \tilde{a}_{j} \epsilon^{2 k+1} j^{2 k+1} \\
& -\sum_{k=1}^{N-1} \tilde{b}_{j} \epsilon^{2 k+1} j^{2 k+1} \\
& e_{N+j}=i \sum_{k=1}^{N-1} \tilde{a}_{j} \epsilon^{2 k+1} j^{2 k+1} \\
& +\sum_{k=1}^{N-1} \tilde{b}_{j} \epsilon^{2 k+1} j^{2 k+1} \\
& 1 \leq j \leq N
\end{aligned}
$$

\section{Quasi-rational solutions of order 12 with twenty two parameters}

We have already explicitly constructed quasi rational solutions to NLS equation from order 2 until 11 . We construct here deformations of the Peregrine breather $P_{12}$ of order 12 depending on 22 parameters. We do not give the analytic expression of the solution to NLS equation of order 12 with twenty two parameters. The computations were done using the computer algebra systems Maple and TRIP [38]. For simplicity, we denote

$$
\begin{aligned}
& d_{3}:=\operatorname{det}\left(\left(n_{j k)_{j, k \in[1,2 N]}}\right),\right. \\
& d_{1}:=\operatorname{det}\left(\left(d_{j k)_{j, k \in[1,2 N]}}\right) .\right.
\end{aligned}
$$

The number of terms of the polynomials of the numerator $d_{3}$ and denominator $d_{1}$ of the solutions are shown in the table below (Table 1) when other $a_{i}$ and $b_{i}$ are set to 0 .

where $\lambda_{j}$ are given for $1 \leq j \leq N$ by :

$$
\lambda_{j}=1-2 j^{2} \epsilon^{2}, \lambda_{N+j}=-\lambda_{j} .
$$

The terms $x_{r, \nu}(r=3,1)$ are defined for $1 \leq \nu \leq 2 N$ by :

$$
x_{r, \nu}=(r-1) \ln \frac{\gamma_{\nu}-i}{\gamma_{\nu}+i} .
$$




\begin{tabular}{|l|r|}
\hline $\mathrm{N}=11$ & number of terms \\
\hline$d 3\left(a_{1}, b_{1}, x, t\right)$ & 1535989 \\
$d 1\left(a_{1}, b_{1}, x, t\right)$ & 777834 \\
$d 3\left(a_{2}, b_{2}, x, t\right)$ & 579793 \\
$d 1\left(a_{2}, b_{2}, x, t\right)$ & 293604 \\
$d 3\left(a_{3}, b_{3}, x, t\right)$ & 310973 \\
$d 1\left(a_{3}, b_{3}, x, t\right)$ & 157500 \\
$d 3\left(a_{4}, b_{4}, x, t\right)$ & 196817 \\
$d 1\left(a_{4}, b_{4}, x, t\right)$ & 99668 \\
$d 3\left(a_{5}, b_{5}, x, t\right)$ & 196817 \\
$d 1\left(a_{5}, b_{5}, x, t\right)$ & 70052 \\
$d 3\left(a_{6}, b_{6}, x, t\right)$ & 138317 \\
$d 1\left(a_{6}, b_{6}, x, t\right)$ & 52513 \\
$d 3\left(a_{7}, b_{7}, x, t\right)$ & 81416 \\
$d 1\left(a_{7}, b_{7}, x, t\right)$ & 41240 \\
$d 3\left(a_{8}, b_{8}, x, t\right)$ & 65909 \\
$d 1\left(a_{8}, b_{8}, x, t\right)$ & 33334 \\
$d 3\left(a_{9}, b_{9}, x, t\right)$ & 52787 \\
$d 1\left(a_{9}, b_{9}, x, t\right)$ & 26728 \\
$d 3\left(a_{10}, b_{10}, x, t\right)$ & 38627 \\
$d 1\left(a_{10}, b_{10}, x, t\right)$ & 19547 \\
$d 3\left(a_{11}, b_{10}, x, t\right)$ & 21558 \\
$d 1\left(a_{11}, b_{10}, x, t\right)$ & 10908 \\
\hline
\end{tabular}

Table 1: Number of terms for the polynomials $d_{3}$ and $d_{1}$ of the solutions of the NLS equation.

We construct figures to show deformations of the Peregrine breather of order 12. We get different types of symmetries in the plots in the $(x, t)$ plane. We give some examples of this fact in the following discussion.

\subsection{Peregrine breather of or- der 12}

If we choose $\tilde{a}_{i}=\tilde{b}_{i}=0$ for $1 \leq i \leq 10$, we obtain the classical eleventh Peregrine breather

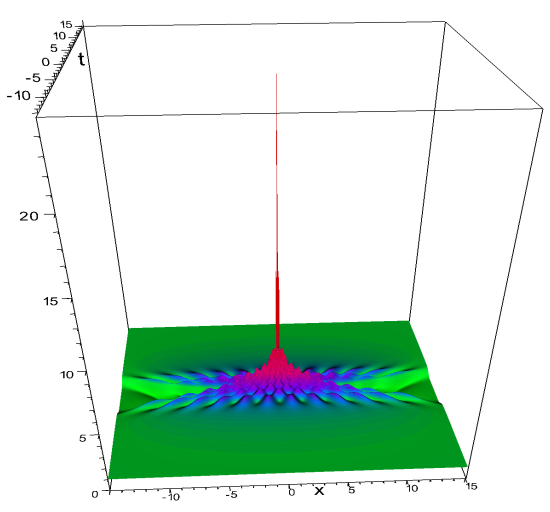

Figure 1: Solution of NLS, N=12, all parameters equal to 0 , Peregrine breather $P_{12}$.

\subsection{Variation of parameters}

With other choices of parameters, we obtain all types of configurations : triangles and multiple concentric rings configurations with a maximum of 78 peaks.
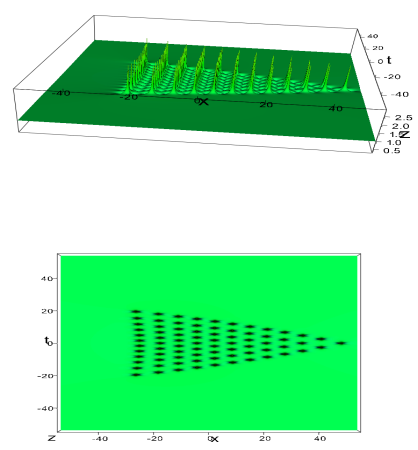

Figure 2: Solution of NLS, N=12, $\tilde{a}_{1}=$ $10^{3}$ : triangle with 78 peaks; on the right, sight from top. 

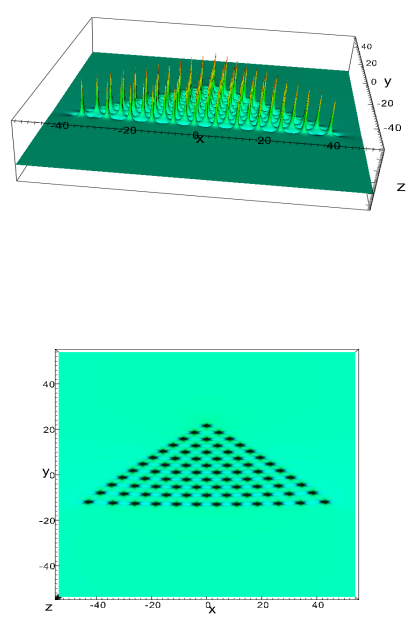

Figure 3: Solution of NLS, N=12, $\tilde{b}_{1}=$ $10^{3}$ : triangle with 78 peaks; on the right, sight from top.
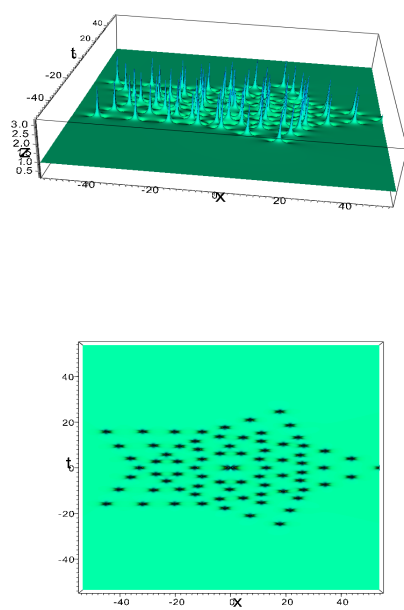

Figure 4: Solution of NLS, N=12, $\tilde{a}_{2}=$ $10^{5}: 7$ rings with $5,10,10,10,20,10$, 10 ,peaks on each of them with in the center $P_{2}$; on the right, sight from top.
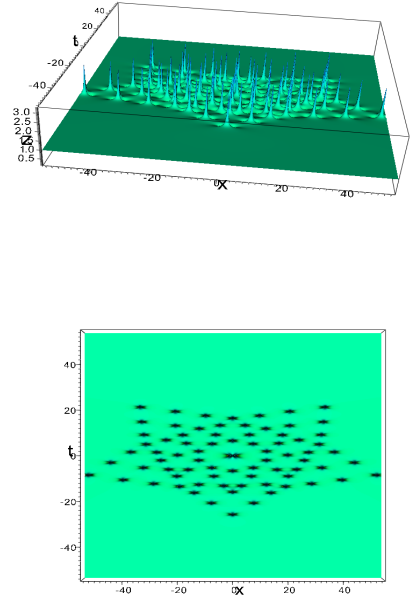

Figure 5: Solution of NLS, N=12, $\tilde{b}_{2}=$ $10^{5}: 7$ rings with $5,10,10,10,20,10$, 10 , peaks on each of them with in the center $P_{2}$; on the right, sight from top.
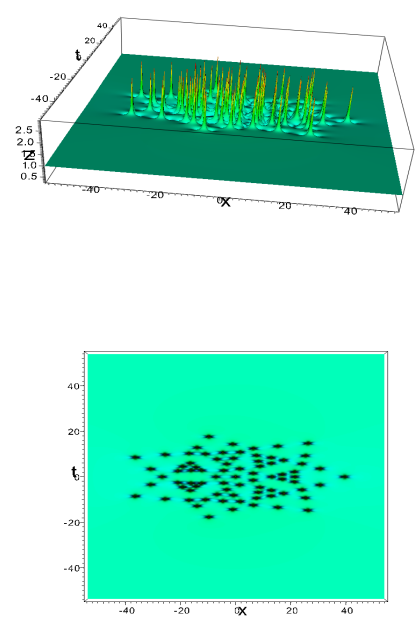

Figure 6: Solution of NLS, N=12, $\tilde{a}_{3}=$ $10^{6}$ : 7 rings with $7,14,7,21,14,7$, 7 peaks with in the center $P_{1}$; on the right, sight from top. 

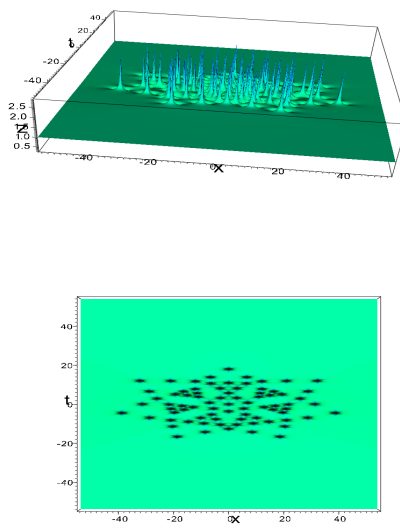

Figure 7: Solution of NLS, N=12, $\tilde{b}_{3}=$ $10^{6}: 7$ rings with $7,14,7,21,14,7$, 7 peaks with in the center $P_{1}$; on the right, sight from top.
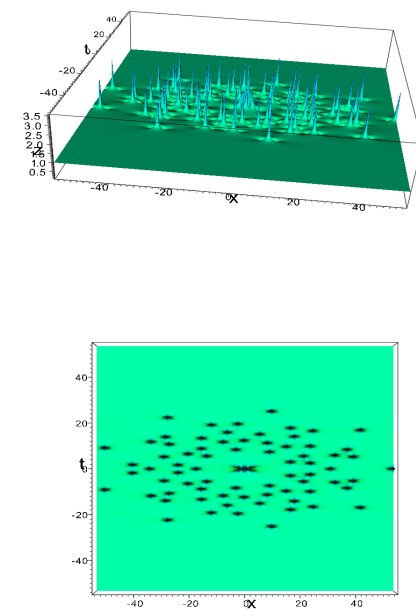

Figure 8: Solution of NLS, N=12, $\tilde{a}_{4}=$ $10^{8}: 6$ rings with $9,18,9,9,19,9$ peaks, in the center $P_{3}$; on the right, sight from top.
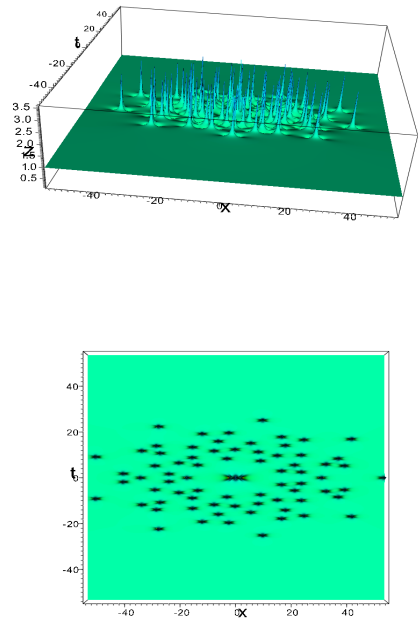

Figure 9: Solution of NLS, N=12, $\tilde{b}_{4}=$ $10^{8}: 6$ rings with $9,18,9,9,19,9$ peaks, in the center $P_{3}$; on the right, sight from top.
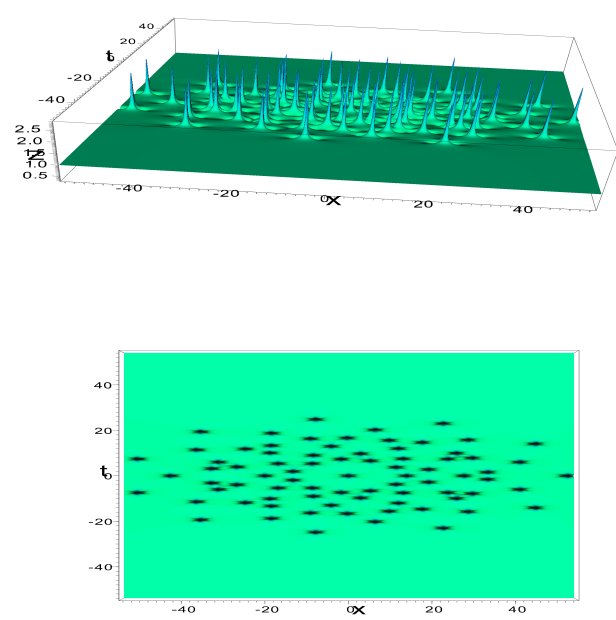

Figure 10: Solution of NLS, N=11, $\tilde{a}_{5}=10^{11}: 6$ rings of $11,11,22,11$, 11,11 peaks one peak in he center; on the right, sight from top. 

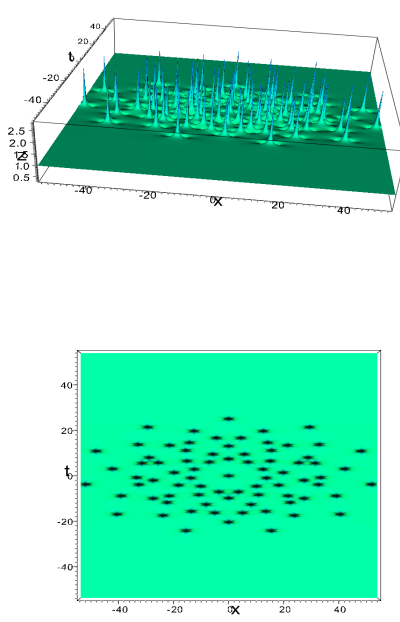

Figure 11: Solution of NLS, N=11, $\tilde{b}_{5}=10^{11}: 6$ rings of $11,11,22,11$, 11,11 peaks one peak in he center; on the right, sight from top.
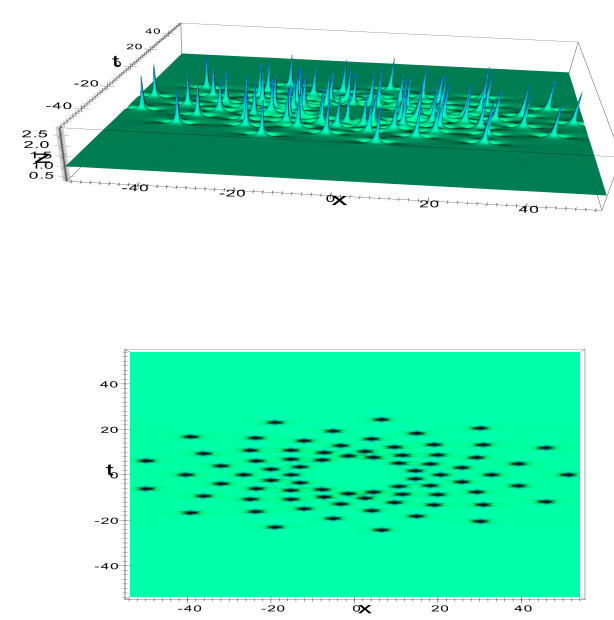

Figure 12: Solution of NLS, N=12, $\tilde{a}_{6}=10^{13}: 6$ rings with 13 peaks without peak in the center; on the right, sight of top.
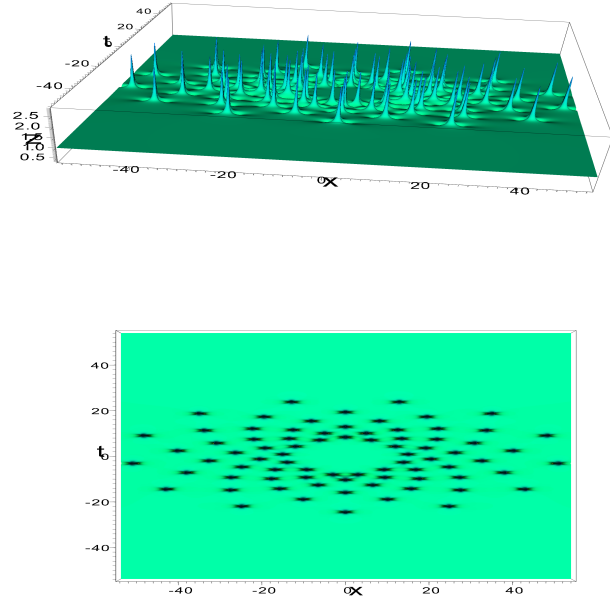

Figure 13: Solution of NLS, N=12, $\tilde{b}_{6}=10^{13}: 6$ rings with 13 peaks without peak in the center; on the right, sight of top.
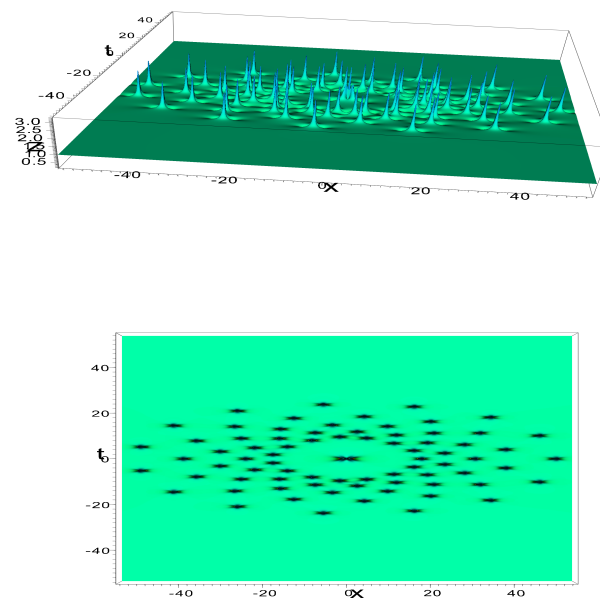

Figure 14: Solution of NLS, N=12, $\tilde{a}_{7}=10^{15}: 5$ rings with 15 peaks and in the center the Peregrine breather of order 2; on the right, sight from top. 

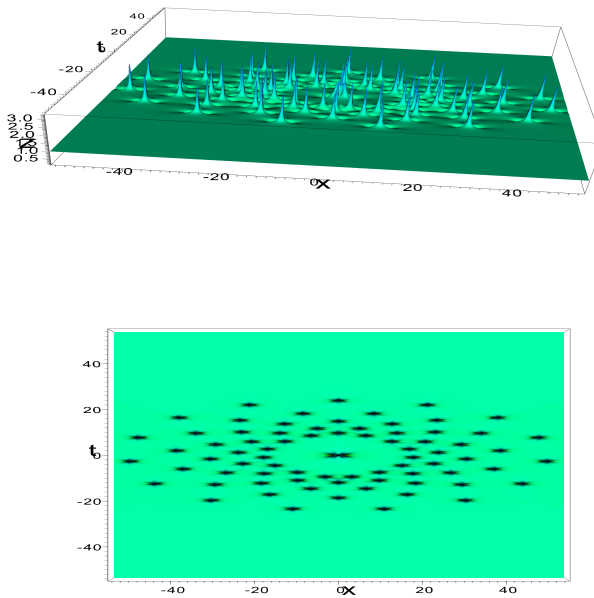

Figure 15: Solution of NLS, N=12, $\tilde{a}_{7}=10^{15}: 5$ rings with 15 peaks and in the center the Peregrine breather of order 2 ; on the right, sight from top.
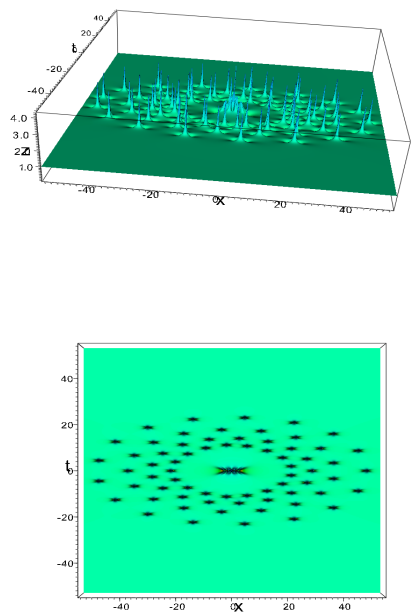

Figure 16: Solution of NLS, N=12, $\tilde{a}_{8}=10^{18}: 4$ rings with 17 peaks and in the center the Peregrine breather of order 4; on the right, sight from top.
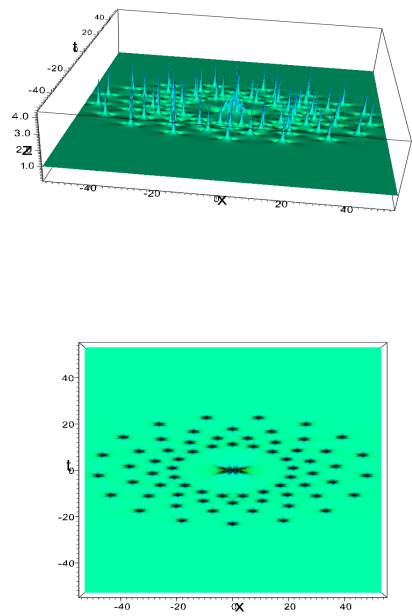

Figure 17: Solution of NLS, N=12, $\tilde{b}_{8}=10^{18}: 4$ rings with 17 peaks and in the center the Peregrine breather of order 4; on the right, sight from top.
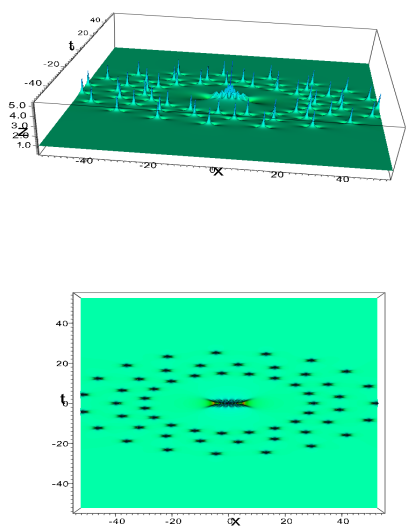

Figure 18: Solution of NLS, N=10, $\tilde{a}_{9}=10^{20}: 3$ rings with 19 peaks and in the center the Peregrine breather of order 6 ; on the right, sight from top. 

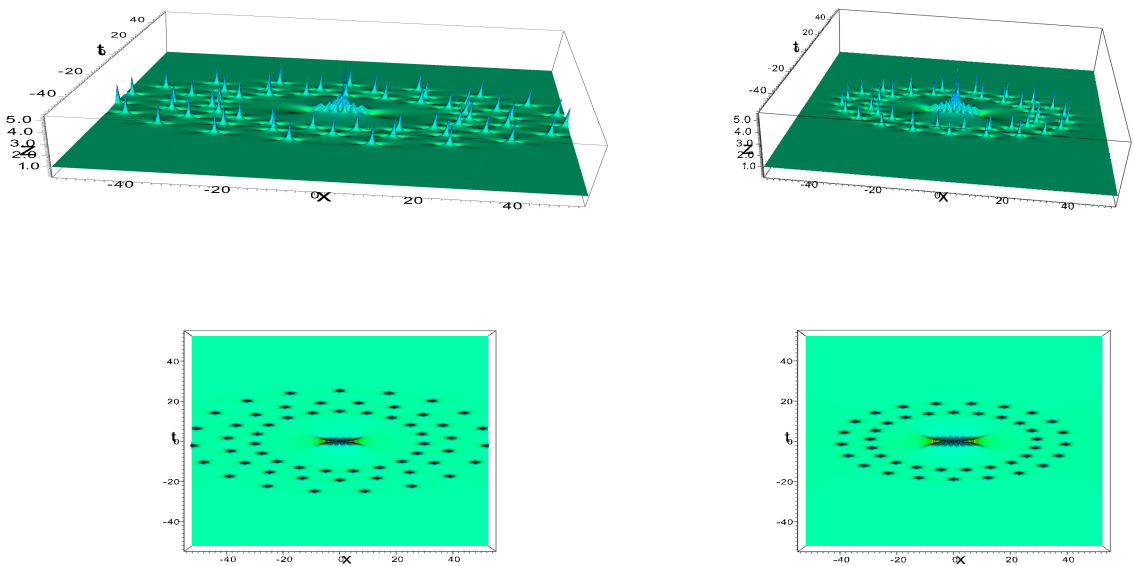

Figure 19: Solution of NLS, N=10, $\tilde{b}_{9}=10^{20}: 3$ rings with 19 peaks and in the center the Peregrine breather of order 6 ; on the right, sight from top.

21: Solution of NLS, $\mathrm{N}=12$, $\tilde{b}_{10}=10^{20}:$ two rings with 21 peaks and in the center the Peregrine breather of order 8 ; on the right, sight from top.
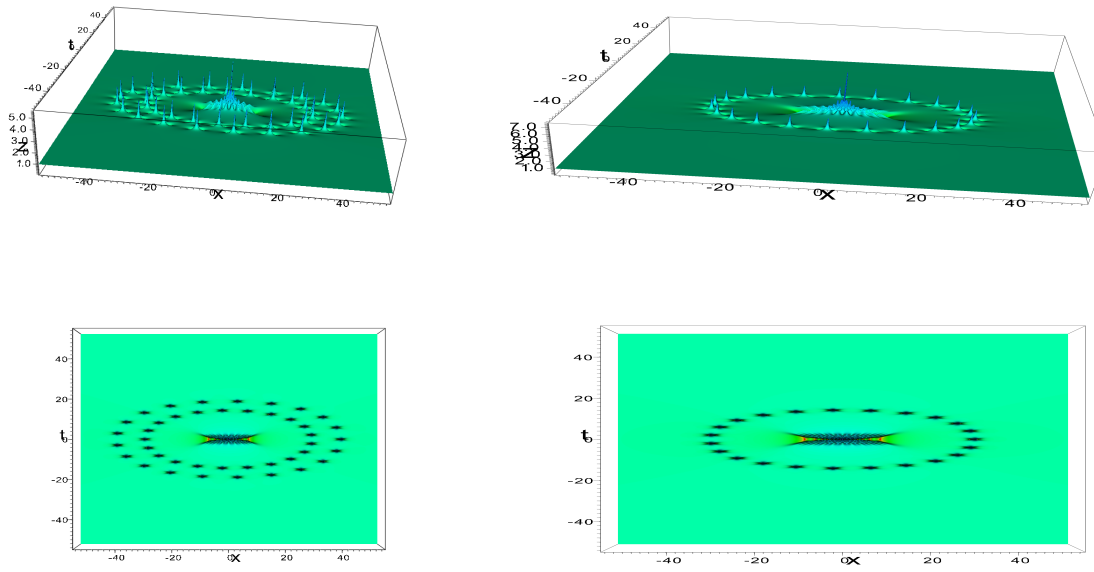

Figure 20: Solution of NLS, N=12, $\tilde{a}_{10}=10^{20}:$ two rings with 21 peaks and in the center the Peregrine breather of order 8 ; on the right, sight from top.

Figure 22: Solution of NLS, N=12, $\tilde{a}_{11}=10^{20}:$ one ring with 23 peaks and in the center the Peregrine breather of order 10; on the right, sight from top. 

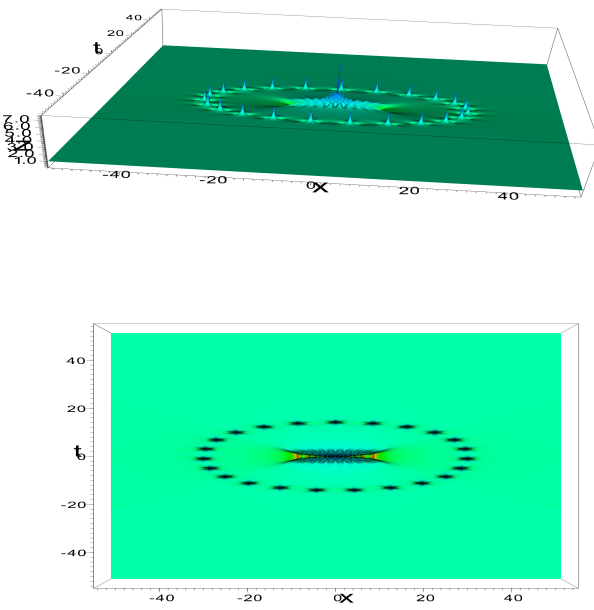

Figure 23: Solution of NLS, N=12, $\tilde{b}_{11}=10^{20}$ : one ring with 23 peaks and in the center the Peregrine breather of order 10; on the right, sight from top.

\section{Conclusion}

Among the various methods currently built to determine explicit solutions of the nonlinear Schrdinger equation, by using the transformations of Darboux $[6,7,39]$, the bilinear method of Hirota [40], the ratio of special determinants $[10,11]$ or the wronskiens $[41,16,32]$, the method presented in this text is the most effective.

We have constructed explicitly multiparametric solutions to the NLS equation of order 12 with 22 real parameters. The explicit representation in terms of polynomials of degree 156 in $x$ and $t$ is obtained. We cannot give its expression because of his length. It confirms the property about the shape of the breather in the $(x, t)$ coordinates, the maximum of amplitude equal to $2 N+1$ and the degree of polynomials in $x$ and $t$ here equal to $N(N+1)$.

We obtained different patterns in the $(x ; t)$ plane by different choices of these parameters. So we obtain a classification of the rogue waves at order 12 .

We obtain two types of patterns : the triangular shape and the concentric rings for the same index $i$ for $a_{i}$ or $b_{i}$ non equals to 0 .

In the cases $a_{1} \neq 0$ or $b_{1} \neq 0$ we obtain triangles with a maximum of 78 peaks; for $a_{2} \neq 0$ or $b_{2} \neq 0$, we have 7 rings with $5,10,10,10,20,10,10$ peaks with in the center the Peregrine $P_{2}$. For $a_{3} \neq 0$ or $b_{3} \neq 0$, we obtain 7 rings with $7,14,7,21,14,7,7$ peaks with in the center one peak. For $a_{4} \neq 0$ or $b_{4} \neq 0$, we have 6 rings with 9,18 , $9,9,18,9$ peaks with in the center the Peregrine $P_{3}$. For $a_{5} \neq 0$ or $b_{5} \neq 0$, we have 6 rings of $11,11,22,11,11$, 11 peaks with in the center, one peak. For $a_{6} \neq 0$ or $b_{6} \neq 0$, we have 6 rings with 13 peaks on each of them without peak in the center. For $a_{7} \neq 0$ or $b_{7} \neq 0$, we have 5 rings with 15 peaks on each of them and in the center the Peregrine breather of order 2. For $a_{8} \neq 0$ or $b_{8} \neq 0$, we have 4 rings with 17 peaks on each of them and in the center the Peregrine breather of order 4 . For $a_{9} \neq 0$ or $b_{9} \neq 0$, we have 3 rings with 19 peaks and in the center the Peregrine breather of order 6 . For $a_{10} \neq 0$ or $b_{10} \neq 0$, we have 2 rings with 21 peaks and in the center the Peregrine breather of order 8. At least, for $a_{11} \neq 0$ or $b_{11} \neq 0$, we have only one ring with 23 peaks and in the center the Peregrine breather of order 10.

With the previous study, we can formulate the following conjectures about the structure of solutions to the NLS equation based on the parameters $a_{j}$ and $b_{j}$; we chose the convention that $P_{0}$ represent 0 peak. These conjectures 
are given under the hypothesis that only one parameter is not equal to 0 :

at order $N$, for $a_{1} \neq 0$ or $b_{1} \neq 0$, the modulus of the solution to the NLS equation presents the configuration of a triangle with $N(N+$ 1) $/ 2$ peaks;

at order $N$, in the case $1 \leq i \leq\left[\frac{N}{2}\right]$, for $a_{N-i} \neq 0$ or $b_{N-i} \neq 0$, the modulus of the solution to the NLS equation presents $i$ concentric rings with $2 N-2 i+1$ peaks and in the center the $P_{N-2 i}$ breather;

at order $N$, in the case $\left[\frac{N}{2}\right]<i \leq$ $N-2$, for $a_{N-i} \neq 0$ or $b_{N-i} \neq 0$, the modulus of the solution to the NLS equation presents $n_{k}$ rings of $k(2 N-2 i+1)$ peaks, for $1 \leq k \leq r$, $\left.r n_{r}(2 N-2 i+1)<N(N+1) / 2\right)$ with in the center the $P_{N-2 i}$ breather, verifying

$\sum_{n_{k}=1}^{r} n_{k} k(2 N-2 i+1)=2 i N-2 i^{2}+i$.

It currently has many applications in various fields like nonlinear optics [42] or hydrodynamics [43]. We hope that this work will be able to lead to physical applications and to bring a better comprehension of the phenomenon of the rogue waves.

It would be relevant to continue this study to try to classify them in the general case of order $N(N>11)$ and to prove the preceding conjectures.

\section{References}

[1] V. E. Zakharov, Stability of periodic waves of finite amplitude on a surface of a deep fluid, J. Appl.

Tech. Phys, V. 9, 86-94, (1968)

[2] V. E. Zakharov, A.B. Shabat Exact theory of two dimensional self focusing and one dimensinal self modulation of waves in nonlinear media, Sov. Phys. JETP, V. 34, 62-69, (1972)

[3] D. Peregrine, Water waves, nonlinear Schrödinger equations and their solutions, J. Austral. Math. Soc. Ser. B, V. 25, 16-43, (1983)

[4] N. Akhmediev, V. Eleonski, N. Kulagin, Generation of periodic trains of picosecond pulses in an optical fiber : exact solutions, Sov. Phys. J.E.T.P., V. 62, 894-899, (1985)

[5] N. Akhmediev, V. Eleonski, N. Kulagin, Exact first order solutions of the nonlinear Schrödinger equation, Th. Math. Phys., V. 72, N. 2, 183-196, (1987)

[6] N. Akhmediev, A. Ankiewicz, J.M. Soto-Crespo, Rogue waves and rational solutions of nonlinear Schrödinger equation, Physical Review E, V. 80, N. 026601, (2009)

[7] N. Akhmediev, A. Ankiewicz, P.A. Clarkson, Rogue waves, rational solutions, the patterns of their zeros and integral relations, J. Phys. A : Math. Theor., V. 43, 122002-1-9, (2010)

[8] P. Gaillard, Families of quasirational solutions of the NLS equation and multi-rogue waves, J. Phys. A : Meth. Theor., V. 44, 1-15, (2011) 
[9] P. Gaillard, Degenerate determinant representation of solution of the NLS equation, higher Peregrine breathers and multi-rogue waves, Jour. Of Math. Phys., V. 54, 013504-1-32, (2013)

[10] L. Ling, L.C. Zhao, Trajectory Characters of rogue waves, arXiv : 1305.5599v1, 24 May, (2013)

[11] A.A. Gelash, V.E. Zakharov, Superregulier solitonic solutions : a novel scenario for the nonlinear stage of modulation instability, Non linearity, V. 27, 1-39, (2014)

[12] P. Gaillard, V.B. Matveev, Wronskian addition formula and its applications, Max-Planck-Institut für Mathematik, MPI 02-31, V. 161, (2002)

[13] P. Gaillard, A new family of deformations of Darboux-Pöschl-Teller potentials, Lett. Math. Phys., V. 68, 77-90, (2004)

[14] P. Gaillard, V.B. Matveev, New formulas for the eigenfunctions of the two-particle Calogero-Moser system, Lett. Math. Phys., V. 89, 1-12, (2009)

[15] P. Gaillard, V.B. Matveev, Wronskian and Casorai determinant representations for DarbouxPöschl-Teller potentials and their difference extensions, J. Phys A : Math. Theor., V. 42, 1-16, (2009)

[16] P. Gaillard, Wronskian representation of solutions of the NLS equation and higher Peregrine breathers, J. Math. Sciences : Adv. Appl., V. 13, N. 2, 71-153, (2012)
[17] P. Gaillard, Wronskian representation of solutions of NLS equation and seventh order rogue waves, J. Mod. Phys., V. 4, N. 4, 246-266, (2013)

[18] P. Gaillard, V.B. Matveev, Wronskian addition formula and Darboux-Pöschl-Teller potentials, J. Math., V. 2013, ID 645752, 1-10, (2013)

[19] P. Gaillard, Two parameters deformations of ninth Peregrine breather solution of the NLS equation and multi rogue waves, J. Math., V. 2013, 1-111, (2013)

[20] P. Gaillard, Two-parameters determinant representation of seventh order rogue waves solutions of the NLS equation, J. Theor. Appl. Phys., V. 7, N. 45, 1-6, (2013)

[21] P. Gaillard, Six-parameters deformations of fourth order Peregrine breather solutions of the NLS equation, J. Math. Phys., V. 54, 073519-1-22, (2013)

[22] P. Gaillard, Deformations of third order Peregrine breather solutions of the NLS equation with four parameters, Phys. Rev. E, V. 88, 042903-1-9, (2013)

[23] P. Gaillard, Ten parameters deformations of the sixth order Peregrine breather solutions of the NLS equation, Phys. Scripta, V. 89, 015004-1-7, (2014)

[24] P. Gaillard, The fifth order Peregrine breather and its eight-parameters deformations solutions of the NLS equation, Commun. Theor. Phys., V. 61, 365-369, (2014) 
[25] P. Gaillard, Higher order Peregrine breathers, their deformations and multi-rogue waves, J. Of Phys. : Conf. Ser., V. 482, 012016-1-7, (2014)

[26] P. Gaillard, M. Gastineau, Eighteen parameter deformations of the Peregrine breather of order ten solutions of the NLS equation, Int. J. Mod. Phys. C, V. 26, N. 2, 1550016-1-14, (2014)

[27] P. Gaillard, Two parameters wronskian representation of solutions of nonlinear Schrödinger equation, eight Peregrine breather and multi-rogue waves, J. Math. Phys., V. 5, 093506-1-12, (2014)

[28] P. Gaillard, Hierarchy of solutions to the NLS equation and multirogue waves, J. Phys. : Conf. Ser., V. 574, 012031-1-5, (2015)

[29] P. Gaillard, Tenth Peregrine breather solution of the NLS, Ann. Phys., V. 355, 293-298, (2015)

[30] P. Gaillard, M. Gastineau, The Peregrine breather of order nine and its deformations with sixteen parameters solutions of the NLS equation Phys. Lett. A, V. 379, 1309-1313, (2015)

[31] P. Gaillard, Other 2N-2 parameters solutions to the NLS equation and $2 \mathrm{~N}+1$ highest amplitude of the modulus of the N-th order AP breather, J. Phys. A: Math. Theor., V. 48, 145203-1-23, (2015)

[32] P. Gaillard, Multi-parametric deformations of the Peregrine breather of order $\mathrm{N}$ solutions to the NLS equation and multi-rogue waves, Adv. Res., V. 4, 346-364, (2015)

[33] P. Gaillard, Higher order Peregrine breathers solutions to the NLS equation, Jour. Phys. : Conf. Ser., V. 633, 012106-1-6, (2015)

[34] P. Gaillard, M. Gastineau Patterns of deformations of Peregrine breather of order 3 and 4, solutions to the NLS equation with multi-parameters, Journal of Theoretical and Applied Physics, (2016)

[35] P. Gaillard, M. Gastineau Twenty parameters families of solutions to the NLS equation and the eleventh Peregrine breather, Commun. Theor. Phys, V. 65, 136-144, (2016)

[36] P. Gaillard, Rational solutions to the KPI equation and multi rogue waves, Annals Of Physics, V. 355, 293-298, (2016)

[37] P. Gaillard, Towards a classification of the quasi rational solutions to the NLS equation, Teoreticheskaya Y Matematicheskaya Fyzyka, (2015)

[38] M. Gastineau, J. Laskar, TRIP 1.3.8 : TRIP Reference manual, IMCCE, Paris Observatory, (2014), http://www.imcce.fr/trip/

[39] B. Guo, L. Ling, Q.P. Liu, Nonlinear Schrodinger equation: Generalized Darboux transformation and rogue wave solutions, Phys. Rev. E, V. 85, 026607, (2012) 
[40] Y. Ohta, J. Yang, General high-order rogue waves and their dynamics in the nonlinear Schrödinger equation, Pro. R. Soc. A, V. 468, 1716-1740, (2012)

[41] P. Dubard, P. Gaillard, C. Klein, V.B. Matveev, On multi-rogue waves solutions of the NLS equation and positon solutions of the KdV equation, Eur. Phys. J. Special Topics, V. 185, 247-258, (2010)

[42] B. Kibler, J. Fatome, C. Finot, G. Millot, F. Dias, G. Genty, N. Akhmediev, J.M. Dudley, The Peregrine soliton in nonlinear fibre optics, Nat, Phys, V. 6, 790795, (2010)

[43] A. Chabchoub, H. Hoffmann, M. Onorato, N. Akhmediev, Super rogue waves: observation of a higher-order breather in water waves, Phys. Review X, V. 2, 16, (2012). 